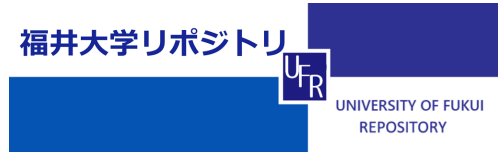

I nvesti gati on of i nf I ammabl e I i qui ds by ter ahertz spect roscopy

\begin{tabular}{|l|l|}
\hline 著者 & $\begin{array}{l}\text { I KEDA Takeshi, MATSUSH TA Aki ra, TATSUNO } \\
\text { M chi aki, M NAM Yuki o, YANAGUCH Mar i ko, } \\
\text { YAMAMOTO Kohj i, TAN Masahi ko, HANGYO Nasanor i }\end{array}$ \\
\hline $\begin{array}{l}\text { j our nal or } \\
\text { publ i cat i on ti tl e }\end{array}$ & Appl i ed physi cs I et ter s \\
\hline vol une & 87 \\
\hline number & 3 \\
\hline page r ange & 34105 \\
\hline year & $2005-07$ \\
\hline URL & ht t p: //hdl . handl e. net /10098/3035 \\
\hline
\end{tabular}




\title{
Investigation of inflammable liquids by terahertz spectroscopy
}

\author{
Takeshi Ikeda, Akira Matsushita, Michiaki Tatsuno, and Yukio Minami \\ Forensic Science Laboratory, Osaka Prefectural Police Headquarters, Honmachi, Chuo-ku, Osaka 541- \\ 0053, Japan
}

\begin{abstract}
Mariko Yamaguchi, Kohji Yamamoto, Masahiko Tani, and Masanori Hangyo ${ }^{\text {a) }}$ Institute of Laser Engineering, Osaka University, 2-6 Yamadaoka, Suita, Osaka 565-0871, Japan
\end{abstract}

(Received 4 January 2005; accepted 6 June 2005; published online 14 July 2005)

\begin{abstract}
We have examined a nondestructive and contactless screening method for water and inflammable liquids stored in common beverage plastic bottles by the terahertz time-domain spectroscopy. No THz transmission has been observed between 10 and $60 \mathrm{~cm}^{-1}$ for $45-\mathrm{mm}$-thick water in plastic bottles. Part of the $\mathrm{THz}$ wave, on the other hand, has been transmitted through the commercially available inflammable liquids in the same bottles. The differences in the absorption intensities and the refractive indices in the $\mathrm{THz}$ region, which reflect the ingredients of organic compounds, allow us to distinguish these inflammable liquids. We confirm that the screening method based on the $\mathrm{THz}$ transmission can be applied to classifying the commercially available inflammable liquids stored in plastic bottles and to distinguishing these inflammable liquids from water as well. (C) 2005 American Institute of Physics. [DOI: 10.1063/1.1999847]
\end{abstract}

During the last two decades, the femtosecond- or picosecond-laser-based methods of generating and detecting pulsed terahertz $(\mathrm{THz})$ electromagnetic waves ${ }^{1}$ has spurred advances in the $\mathrm{THz}$ spectroscopic studies on liquids, 2,3 vapor, ${ }^{4}$ polymers, ${ }^{5}$ and biomolecules. ${ }^{6-9}$ The ultrashort laserbased $\mathrm{THz}$ spectroscopy, named as $\mathrm{THz}$ time-domain spectroscopy (THz-TDS), enables us to deduce the spectra of the refractive index as well as that of the absorption from the phase and amplitude of time-domain signals with a high signal-to-noise ratio. $\mathrm{THz}$ waves penetrate partly through popular packing materials such as paper, ${ }^{10,11}$ polymer, ${ }^{12}$ clothes, ${ }^{13}$ and wood, ${ }^{14}$ allowing us to take sight-through $\mathrm{THz}$ spectra of objects concealed with these visually opaque packing materials and clothes. Materials have their intrinsic $\mathrm{THz}$ spectra in the absorption and the refractive index, usually called as "fingerprint" THz spectra, bands of which originate in intermolecular and intramolecular vibrations. For example, plastic explosives have been shown to be identified by their fingerprint $\mathrm{THz}$ spectra. ${ }^{11,15,16}$ Inspection based on the THz-TDS, therefore, can potentially be applied to screening and identification of sealed-off objects.

The development of inspection methods for hand-carried plastic bottles containing liquids has been the urgent issue on the security in subways, in airplanes, at airports and at stadiums. Inflammable liquids such as gasoline can be carried around by being stored in plastic containers. These bottled liquids have been possible threats of attacks by arsonists and terrorists, who ignite them in closed spaces full of passengers. Inflammable liquids, however, cannot presently be distinguished from water with ease when they are concealed in commonly used beverage plastic bottles. Mid-IR absorption and Raman scattering are possible methods of inspecting inflammable liquids stored in transparent bottles. These spectroscopic techniques, however, cannot be applied to opaque plastic bottles. It has been shown that several gasoline samples have many low-frequency vibrational absorption

\footnotetext{
a) Author to whom correspondence should be addressed; electronic mail:
} hangyo@ile.osaka-u.ac.jp bands from 5 to $650 \mathrm{~cm}^{-1}$, which were measured by Fouriertransform far-infrared spectrometer and the THz-TDS. ${ }^{17}$ The purpose of this letter is to examine a nondestructive and contactless screening method using the THz-TDS for liquids stored in popular plastic bottles made of polyethylene (PE) and polyethylene terephthalate (PET). The transmittances and the refractive indices in the $\mathrm{THz}$ region are dependent on the kinds of commercially available inflammable liquids, allowing us to distinguish these liquids even in the plastic bottles. Water, which does not transmit the $\mathrm{THz}$ waves, can be easily distinguished from the other inflammable liquids by the criterion whether a $\mathrm{THz}$ wave is transmitted or not. The method of the $\mathrm{THz}$ screening, which employs the nonionizing radiation, can be conducted safely to human bodies as well as without any chemical reactions and changes in test objects.

We carried out the THz-TDS with a standard system (Fig. 1) using photoconductive emitter and detector antennas. A mode-locked Ti:sapphire laser (Tsunami, Spectra Physics Ltd.) with a $50 \mathrm{fs}$ pulse width pumped the antennas for generating and detecting pulsed $\mathrm{THz}$ waves. $\mathrm{THz}$ transmission

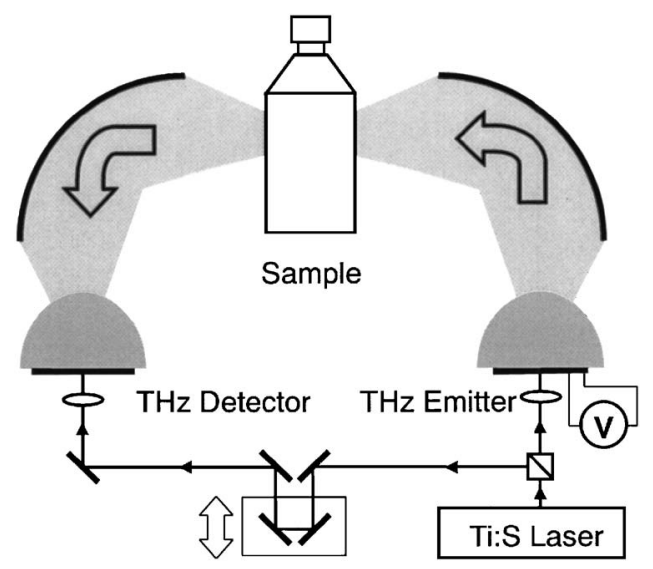

FIG. 1. Schematic diagram of the transmission measurement of the THzTDS. A plastic bottle containing a liquid sample is placed in the path of the THz wave. 


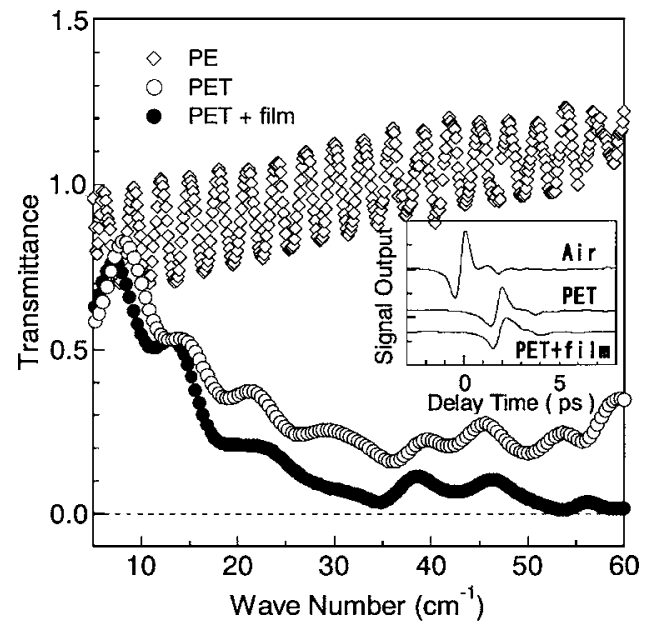

FIG. 2. THz transmittance of the plastic bottles. Inset: temporal behavior of the THz pulse transmitted through air, the PET bottles with and without a plastic label.

measurements were conducted for a liquid sample stored in a plastic bottle in the path of the $\mathrm{THz}$ wave. Commercially available inflammable liquid samples, i.e., gasoline (flash point (fp): $\sim-40{ }^{\circ} \mathrm{C}$ ), benzine (fp: $\sim-40{ }^{\circ} \mathrm{C}$ ), kerosene (fp: $\sim 40{ }^{\circ} \mathrm{C}$ ), and gas oil (fp: $\sim 50{ }^{\circ} \mathrm{C}$ ) were measured. Pure organic solvents of $n$-alkanes $\left(\mathrm{C}_{6} \mathrm{H}_{14}\right.$ and $\left.\mathrm{C}_{8} \mathrm{H}_{18}\right)$ and toluene were also measured for comparison. The PE cylindrical bottles and the PET square bottles were 1 and $0.5 \mathrm{~mm}$ in plastic thickness, and 45 and $60 \mathrm{~mm}$ in path length, respectively. The PET bottles examined in the experiments are generally used as beverage bottles. Accurate $\mathrm{THz}$ measurements for the refractive index were conducted for liquid samples in a stainless-steel cell $(25 \mathrm{~mm}$ in path length for liquid samples except for toluene and $10 \mathrm{~mm}$ in path length for toluene) with silicon windows ( $3 \mathrm{~mm}$ in thickness).

Figure 2 shows the $\mathrm{THz}$ transmittance spectra of the empty PE and PET bottles. The periodic modulations of the spectra are due to the multiple reflections of the $\mathrm{THz}$ waves inside the plastic walls of the bottle. The transmittance of the PE bottle in the THz region is larger than that of the PET bottle although the wall of the former is thicker than that of the latter. The increase of polar groups in polymers has been suggested to increase the absorption intensity in the $\mathrm{THz}$ region. ${ }^{5}$ Ketone, which has the polar keto group $(>\mathrm{C}=\mathrm{O})$ binding two hydrocardon radicals, has been predicted to have resonance absorption between 0.3 and $3 \mathrm{THz}$ in solid state. ${ }^{18}$ The polar carbonyl groups in the PET chains are, therefore, considered to contribute to the increase in the $\mathrm{THz}$ absorption, leading to the low transmittance in the $\mathrm{THz}$ region. The $\mathrm{THz}$ radiation between 5 and $60 \mathrm{~cm}^{-1}$ is, nonetheless, transmitted through the PET bottle sufficiently to conduct the inspection of concealed liquids. The inset of Fig. 2 represents the influence of the plastic label on the temporal behavior of the THz pulses. The transmission through the colored and optically opaque plastic label film over the PET bottle reduces the peak intensity of the $\mathrm{THz}$ pulse, resulting in the low transmittance in Fig. 2. A considerable portion of the $\mathrm{THz}$ pulse can, however, be transmitted through both label and PET bottle. This result shows the feasibility of the THz inspection through the PET bottle irrespective of the opaque plastic label over it. The transmittance larger than unity is observed in the transmittance spectra of the PE bottle. It probably results from the tighter focusing of the transmitted

Downloaded 07 Sep 2005 to 133.1.68.45. Redistribution subject
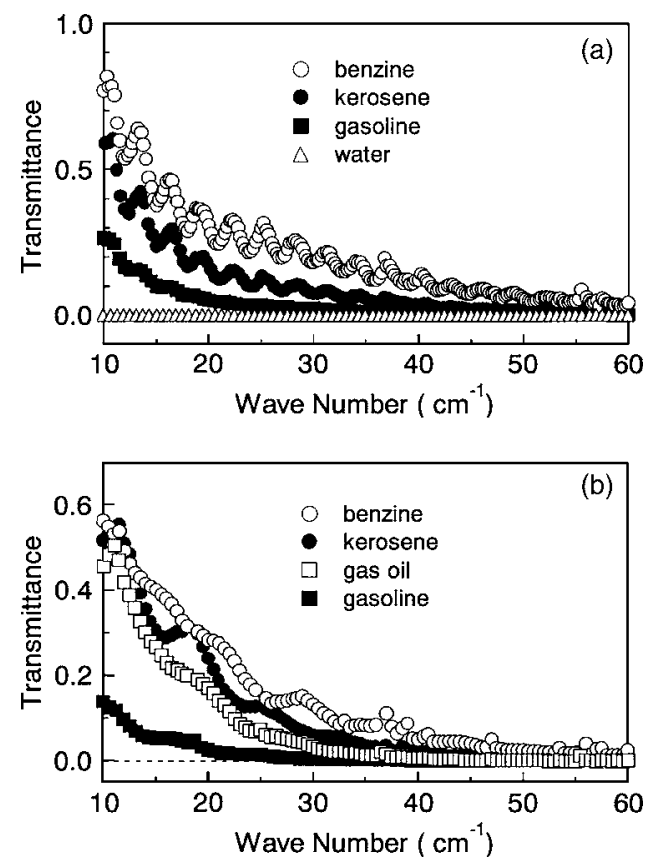

FIG. 3. THz transmittance of the liquids stored in the PE bottle (a) and the PET bottle (b).

$\mathrm{THz}$ wave at the detector antenna than the reference $\mathrm{THz}$ wave due to the lens effect of the "cylindrical" bottle.

Figure 3 represents the $\mathrm{THz}$ transmittance spectra $\left(=\left|E_{b+s}(\widetilde{\nu})\right|^{2} /\left|E_{b}(\widetilde{\nu})\right|^{2}\right)$ of the liquids stored in the PE and PET bottles where $E_{b}(\widetilde{\nu})$ and $E_{b+s}(\widetilde{\nu})$ are complex $\mathrm{THz}$ fields transmitted through an empty bottle and a liquid-containing bottle at a frequency $\widetilde{\nu}$, respectively. Water is highly absorptive in the $\mathrm{THz}$ region, resulting in no $\mathrm{THz}$ transmission for 45-mm-thick water as shown in Fig. 3(a). The inflammable liquids, on the other hand, partly transmit the $\mathrm{THz}$ waves, the transmittance of which is dependent on the species of the liquids. This obvious difference in the THz transmittance allows us to distinguish water from the other liquids very easily. The difference in the $\mathrm{THz}$ transmittance spectra for various liquids also implies the possibility of classification of the inflammable liquids by the THz-TDS. The inflammable liquids with $6 \mathrm{~cm}$ thickness in the PET bottles also partly transmit the THz wave [Fig. 3(b)], indicating that this screening method can be applied to liquids stored in the PET bottle. The periodic modulations on the transmittance spectra in Fig. 3 are due to the multiple reflections as in Fig. 2. Figure 4 represents the $\mathrm{THz}$ absorption spectra of the inflammable liquids, which are obtained from the transmittance spectra in Fig. 3(b). Absorption intensity increases in the order of benzine, kerosene, gas oil, and gasoline. These liquids consist of different kinds of hydrocarbon ingredients, leading to the different intensities of the $\mathrm{THz}$ absorption. The principal ingredient of benzine, kerosene, and gas oil is paraffin (carbon number: 6-24), the absorption of which is very low in the $\mathrm{THz}$ region (absorption coefficient: $0.12-0.14 \mathrm{~cm}^{-1}$ at $35 \mathrm{~cm}^{-1}$ ). Gasoline contains appreciable quantities of aromatic hydrocarbons such as toluene, ethylbenzene, and xylene as the main ingredients ( $\sim 25 \%$ in total), moderately absorbing the $\mathrm{THz}$ wave (absorption coefficient: $0.87-1.48 \mathrm{~cm}^{-1}$ at $35 \mathrm{~cm}^{-1}$ ) owing to the high polarizability of electrons in the aromatic $\pi$ orbitals. ${ }^{2}$ Therefore benzine, kerosene, and gas oil, which contain very small quantities of AIP license or copyright, see http://apl.aip.org/apl/copyright.jsp 


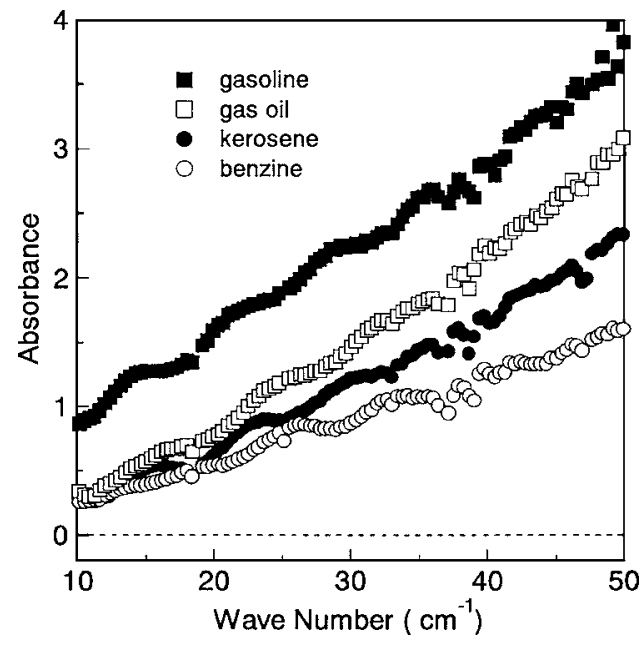

FIG. 4. THz absorption spectra of inflammable liquids in the PET bottles.

aromatic hydrocarbons, show the low $\mathrm{THz}$ absorption intensity relative to that of gasoline.

Figure 5 shows the refractive indices of the inflammable liquids measured by using the stainless steel sample cells with the silicon windows. The refractive indices are distributed from 1.37 to 1.50 with the highest value for toluene and the lowest value for $n-\mathrm{C}_{6} \mathrm{H}_{14}$. The refractive indices of respective inflammable liquids are separated by more than 0.01 with each other. The difference of 0.01 in the refractive index corresponds to the delay of the main $\mathrm{THz}$ pulse by as much as 2 ps after transmitting through a 60 -mm-thick medium (the same thickness of the PET bottles in this study). The delay time of a $\mathrm{THz}$ pulse after transmitted through a 60$\mathrm{mm}$-thick medium with the refractive index of 1.45 , for ex-

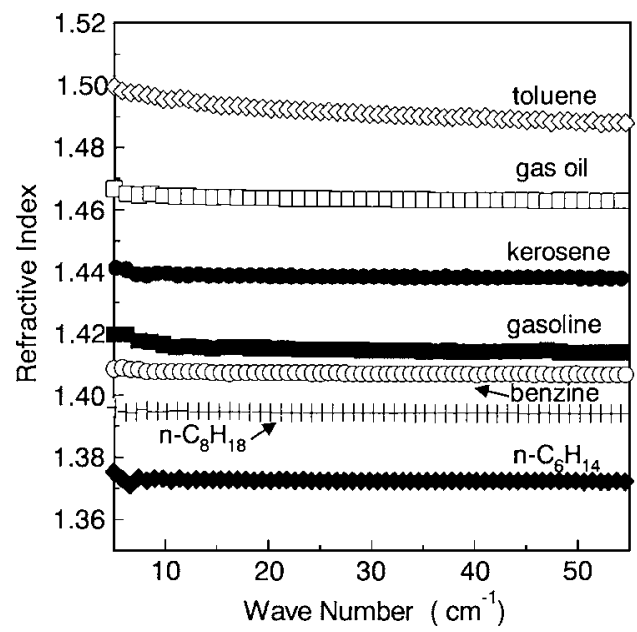

FIG. 5. Refractive indices of the inflammable liquids and pure hydrocarbon solvents. ample, is 90 ps relative to the reference $\mathrm{THz}$ pulse. The time deviation of 2 from 90 ps can be easily detected in the THzTDS. The difference in the refractive indices larger than 0.01 in Fig. 5, therefore, implies that the screening of these liquids can be conducted by detecting an arrival time of a $\mathrm{THz}$ pulse in combination with precise measurement of the sample thickness by such as laser interferometers.

In the present work, we have examined the feasibility of application of the THz-TDS to the nondestructive and contactless screening of liquids stored in the PE and PET bottles. These plastic bottles transmit sufficient amounts of $\mathrm{THz}$ waves to inspect concealed liquids in the bottles. Water and the commercially available inflammable liquids in the bottles can be easily distinguished by the criterion whether a $\mathrm{THz}$ wave is transmitted or not. The differences in the transmittances and the refractive indices in the $\mathrm{THz}$ region imply that the THz-TDS can be used for screening and discrimination of the inflammable liquids.

This work was partially supported by the Grant-in-Aid for Scientific Research from the Japan Society for the Promotion of Science.

${ }^{1}$ M. C. Nuss and J. Orenstein, in Millimeter and Submillimeter Wave Spectroscopy of Solids, edited by G. Grüner (Springer, Berlin, 1998), pp. 7-50.

${ }^{2}$ J. E. Pedersen and S. R. Keiding, IEEE J. Quantum Electron. 28, 2518 (1992).

${ }^{3}$ J. T. Kindt and C. A. Schmuttenmaer, J. Phys. Chem. 100, 10373 (1996).

${ }^{4}$ H. Harde and D. Grischkowsky, J. Opt. Soc. Am. B 8, 1642 (1991).

${ }^{5}$ K. Yamamoto, M. Yamaguchi, M. Tani, M. Hangyo, S. Teramura, T. Isu, and N. Tomita, Appl. Phys. Lett. 85, 5194 (2004).

${ }^{6}$ M. Walther, B. Fischer, M. Schall, H. Helm, and P. U. Jepsen, Chem. Phys. Lett. 332, 389 (2000).

${ }^{7}$ B. M. Fischer, M. Walther, and P. U. Jepsen, Phys. Med. Biol. 47, 3807 (2002).

${ }^{8}$ Y. C. Shen, P. C. Upadhya, E. H. Linfield, and A. G. Davies, Appl. Phys. Lett. 82, 2350 (2003).

${ }^{9}$ M. Yamaguchi, F. Miyamaru K. Yamamoto, M. Tani, and M. Hangyo, Appl. Phys. Lett. 86, 053903 (2005).

${ }^{10}$ K. Kawase, Y. Ogawa, and Y. Watanabe, Opt. Express 11, 2549 (2003).

${ }^{11}$ K. Yamamoto, M. Yamaguchi, F. Miyamaru, M. Tani, M. Hangyo, T. Ikeda, A. Matsushita, K. Koide, M. Tatsuno, and Y. Minami, Jpn. J. Appl. Phys., Part 2 43, L414 (2004).

${ }^{12}$ B. B. Hu and M. C. Nuss, Opt. Lett. 20, 1716 (1995).

${ }^{13}$ J. E. Bjarnason, T. L. J. Chan, A. W. M. Lee, M. A. Celis, and E. R. Brown, Appl. Phys. Lett. 85, 519 (2004).

${ }^{14}$ M. Koch, in Sensing with Terahertz Radiation, edited by D. Mittleman (Springer, Berlin, 2003), pp. 295-316.

${ }^{15}$ M. C. Kemp, P. F. Taday, B. E. Cole, J. A. Cluff, A. J. Fitzgerald, and W. R. Tribe, Proc. SPIE 5070, 17 (2003).

${ }^{16}$ F. Huang, B. Schulkin, H. Altan, J. F. Federici, D. Gary, R. Barat, D. Zimdars, M. Chen, and D. B. Tanner, Appl. Phys. Lett. 85, 5535 (2004).

${ }^{17} \mathrm{~F}$. M. Al-Douseri, C. Yunqing, and, X.-C. Zhang, in CLEO/IQEC (Conference on Lasers and Electro-Optics/ International Quantum Electronics Conference), 2004 OSA Technical Digest (Optical Society of America, San Francisco), Paper No. CMG6.

${ }^{18} \mathrm{H}$. Fröhlich, Theory of Dielectrics, 2nd ed. (Clarendon, Oxford, 1986). 